\title{
Sprache und Kontext
}

\author{
Wolfgang Klein und Willem J.M. Levelt \\ Projektgruppe für Psycholinguistik der Max-Planck-Gesellschaft, Nijmegen, Niederlande
}

Recently, the Max Planck Society founded a new Project group for Psycholinguistics. This article reviews some of the kernel issues of the group's research program. The central concern is with the context dependency of the speaker's linguistic behavior. The process of linguistically formulating depends not only on what the speaker wants to express, but also on what has been said previously (linguistic context), and on the physical and social situation (nonlinguistic context). Special attention is paid to two contextdependent phenomena.

1977 hat die Max-Planck-Gesellschaft in Nijmegen (Niederlande) eine Projektgruppe für Psycholinguistik eingerichtet. Ihr gehören zehn hauptamtliche wissenschaftliche und etwa zwanzig weitere Mitarbeiter an; sie soll zunächst fünf Jahre tätig sein. Die MPG ist damit räumlich und inhaltlich über ihren bisherigen Förderungsbereich hinausgegangen: Weder Psychologie noch Linguistik gehörten bislang zu den von ihr betreuten Gebieten. Dieser Beitrag soll einen Einblick in die Untersuchungen des Institutes geben. Die Absicht ist, eine Vorstellung von den spezifischen Problemen zu geben, um die es in diesem Projekt und damit allgemein in der Psycholinguistik geht.

\section{Sprache und Kontext als psycholinguistisches Forschungsproblem}

Weder Linguistik noch Psychologie pflegen zu den Naturwissenschaften gerechnet zu werden; man wird es daher auch nicht für ihrer beider Sproß, die Psycholinguistik, erwarten können. Während sich aber die Psychologie seit einem Jahrhundert weithin kontrollierter experimenteller Verfahren bedient und damit den Naturwissenschaften zumindest methodisch nicht ganz fern steht, kann man dies für die Linguistik - oder, wie man früher sagte, die Sprachwissenschaft - weder vom Gegenstand noch von der Methode her sagen. Ihr Gegenstand, die Sprache, ist nach einem Wort eines ihrer einflußreichsten Vertreter, Ferdinands de Saussure, ein „fait social" und nicht ein ,fait naturel", und methodisch war sie eher auf die Ermittlung einzelner Fakten und eventuell ihres historischen Zusammenhangs als auf die allgemeiner Gesetzlichkeiten des sprachlichen Verhaltens gerichtet. Dieses - natürlich grob vereinfachte - Bild hat sich in den letzten beiden Jahrzehnten beträchtlich verschoben:

1. Seit etwa Mitte der Fünfzigerjahre wurden in starkem Maße formale Methoden in die Linguistik eingeführt. Weitaus am einflußreichsten war hier das Werk Noam Chomskys und seiner Mitarbeiter, das über den Rahmen der eigentlichen Sprachtheorie, der generativen Transformationsgrammatik, hinaus in eine Reihe anderer Disziplinen hineinwirkte - etwa in die Philosophie (Begriff der Kompetenz, der angeborenen Ideen in Verbindung mit sprachlichen Universalien), in die Mathematik (Theorie der formalen Sprachen), in die Psychologie (z.B. Problem der mentalen Realität grammatischer Strukturen), in Spuren sogar in so fernstehende wie die Biochemie (vgl. z.B. [3]).

2. Die Arbeiten Chomskys und anderer - in jüngerer Zeit vor allem die von Logikern wie Richard Montague - führten in der Linguistik selbst zu einer gewissen Theorielastigkeit; die empirische Erforschung sprachlicher Strukturen und sprachlicher Verhaltensweisen rückte zeitweilig etwas ins zweite Glied. Seit einigen Jahren kehrt sich diese Entwicklung um. Man versucht, die nunmehr verfügbaren formalen Beschreibungsmittel auf die Analyse größerer Datenmengen, die in sehr unterschiedlichen Weisen beschafft werden, anzuwenden. Paradigmatisch dafür sind beispielsweise die Arbeiten des US-Soziolinguisten William Labov. 
Dieser Entwicklung, die keineswegs abgeschlossen ist, folgt auch die neuere Psycholinguistik. Es geht dabei um Probleme wie

Sprache und Wahrnehmung,

Sprache und Kognition,

Verarbeitung von Sprache im Gehirn,

Sprachstörungen,

biologische Grundlagen der Sprache und der Sprachentstehung,

Sprachentwicklung beim Kind.

Charakteristisch für diese Problembereiche ist, daß es in ihnen nicht um die Analyse isolierter sprachlicher Strukturen geht, sondern um Gesetzlichkeiten des sprachlichen Verhaltens und ihren Zusammenhang mit psychologischen, biologischen, medizinischen, aber auch sozialen Faktoren.

Eines der wichtigsten Momente im sprachlichen Verhalten des Menschen ist, daß die sprachlichen Äußerungen selbst in einer Äußerungssituation eng mit Faktoren wie Wahrnehmung, Vorwissen von Sprecher und Hörer, Erwartungen hinsichtlich dessen, was man sinnvollerweise erwarten kann, u.v.a. zusammenhängt. Sprachliches Verhalten ist an den jeweiligen Redekontext gebunden. Dieser Redekontext kann einesteils ganz außersprachlich sein; dies gilt für Kontextfaktoren wie Wahrnehmung, Vorwissen usw. Er kann aber auch sprachlich vermittelt werden; so bilden zu einer gegebenen sprachlichen Äußerung die vorausgehenden und die folgenden jeweils einen „sprachlichen Kontext", ohne den die betreffende Äußerung oft nicht sinnvoll zu interpretieren ist. Die Äußerung ,Ludwig Maria, Fritz Ilse“ erhält - in einer bestimmten Redesituation - ihren Sinn erst dadurch, da $B$ jemand anders zuvor gefragt hat ,Wer hat wen geheiratet?". Ebenso kann man in einer Redesituation eine Äußerung wie "Die da, bitte" nur verstehen, wenn man noch über Möglichkeiten der visuellen Wahrnehmung (etwa einer Zeigegeste) und die Kenntnis bestimmter sozialer Verhaltensweisen verfügt: Wenn jemand beim Metzger auf eine Wurst zeigt und dies (nämlich das obige) sagt, so versteht der Metzger üblicherweise diese Äußerung im Sinne von „Geben Sie mir diese Wurst da, bitte“ o.ä. Was er versteht, geht aufgrund seiner Wahrnehmung und seines Vorwissens über das hinaus, was in Wörtern gesagt wird. In anderem Redezusammenhang kann die Äußerung „Die da, bitte“ etwas ganz anderes bedeuten. Wenn man nur diese Äußerung selbst analysiert, nicht aber, wie sie mit den verschiedenen Kontextfaktoren zusammenspielt, wird man kein angemessenes Bild von der Funktion der Sprache gewinnen können.

Daß die Sprache stark mit dem Kontext zusammenwirkt, ist bekannt und oft betont worden. Für die Psycholinguistik ergibt sich nun die Aufgabe zu klä- ren, wie dies in concreto passiert. Dazu ist mindestens zweierlei erforderlich: Es muß ermittelt werden, an welchen Stellen die sprachlichen Strukturen für den Kontext ,,sensibel“" sind und wie die einzelnen kontextuellen Faktoren das, was die Äußerung selbst offengelassen hat, gleichsam auffüllen.

Die beiden wichtigsten strukturellen Eigenschaften natürlicher Sprachen, an denen der Kontext interveniert, sind Deixis und Ellipse. Deiktika sind Wörter wie ich, da, hier, jetzt, heute, links usw. Das Wort „heute" zum Beispiel hat täglich eine andere Bedeutung, je nachdem, an welchem Tag es gesagt wird. Man kann also sagen, daß morgen heute gestern ist. Worauf , ich" verweist, hängt davon $a b$, wer gerade spricht. $\mathrm{Ob}$ eine Kirche links oder rechts steht, hängt von der Blickrichtung des Sprechers ab, usw. Ellipsen sind Weglassungen, die man regelhaft aus dem Kontext ergänzen kann, z.B. ,(Fritz war gestern abend) im Kino" als Antwort auf die Frage „Wo war Fritz gestern abend ?", oder als zweiter Teil einer Koordination: „Der Vater gibt seiner Frau einen Kuß und der Onkel (gibt seiner Frau) eine Ohrfeige", usw. Im folgenden werden erste Ergebnisse von Pilotstudien im Nijmegener Projekt skizziert. Technische Details sind weggelassen.

\section{Deixis}

Man unterscheidet gewöhnlich zwischen personaler Deixis, z.B. ,ich, du“", temporaler Deixis, z.B. „,nun, vorhin, morgen", und lokaler Deixis, z.B. „,hier, dort", oder, wie bei Karl Valentin (,Tingeltangel"), „drenten“ und ,herenten“. Die Unterscheidung ist etwas grob, weil sie verschiedene Deiktika nicht oder nur in manchen Verwendungsweisen berücksichtigt, wie etwa die Partikel ,,das", und die fließenden Übergänge von einer Kategorie zu einer anderen nicht in Rechnung stellt: So wird das Wort „,da" im Deutschen gleichermaßen temporal wie lokal verwendet (,da kam die böse Fee herein“, ,,da lag er"); man kann sie aber als Arbeitsgrundlage nehmen. Unter diesen Formen der Deixis ist die lokale bislang am wenigsten untersucht, obwohl sie sicher nicht minder wichtig ist als die andern und obwohl sie sich z.B. für empirische Studien eher anbietet als die temporale. Lokaldeiktische Ausdrücke beziehen sich vorwiegend auf Einheiten der wahrnehmbaren (oder erinnerbaren) räumlichen Umgebung; sie sind der Erforschung leichter zugänglich als Ausdrücke, die auf Einheiten unserer ,,inneren Anschauung" verweisen. Die Ursache liegt vielleicht in der außerordentlichen Komplexität des lokaldeiktischen Systems. Um davon einen Eindruck zu vermitteln, betrachten wir im folgenden einige der Faktoren, die den Gebrauch von 
„hier“, ,,dort"“, ,da“ (im lokalen Sinn) im Deutschen mitbestimmen.

\section{Probleme der lokalen Deixis}

Ein Sprecher, der in einer Redesituation Ausdrücke wie ,hier" oder ,dort" oder ,da" verwendet, bezieht sich damit auf bestimmte Einheiten der Außenwelt oder der Vorstellung, auf Denotate dieser Ausdrücke. Diese Denotate sind sehr variabel. Wenn jemand von München nach Köln telefoniert und sagt: ,Hier regnet es", so bedeutet „hier" soviel wie ,in München“ oder ,,in der Gegend von München"; wenn der andre sagt „Hier scheint die Sonne“, so widerspricht er damit nicht, sondern er meint mit "hier" soviel wie ,,in Köln" oder , in der Gegend von Köln“. Beider "hier" hat also ein anderes Denotat, aber beide verstehen jeweils des andern ,hier" : Sie können identifizieren, was jeweils mit, ,hier" gemeint ist. Um zu klären, was sie dazu befähigt, muß man folgende Probleme klären: das der deiktischen Räume, der OrigoMarkierung, der Koordination, der Abgrenzung, der deiktischen Oppositionen und der Analogon-Einführung.

Die Denotate von Wörtern wie „Karl, John, Maria“ sind Personen, nämlich Karl, John und Maria, die Denotate von Wörtern wie ,lachen“, „tanzen“, „,brüllen" sind Handlungen, nämlich lachen, tanzen und brüllen; die Denotate von ,hier", ,dort", ,da" sind Teilräume bestimmter deiktischer Räume. Ein solcher Raum kann z.B. der Raum der visuellen Wahrnehmung sein: „Hier sitzt man ganz gemütlich“. „Hier" verweist in diesem Beispiel vielleicht auf eine Zimmerecke. Es kann sich aber auch um jeden diffusen Raum handeln, der in unserer Vorstellung durch unsere geographischen Kenntnisse gegeben ist, wie in „Hier lebt man billiger als in den Staaten“, oder gar um einen noch wesentlich abstrakteren Raum, der gleichfalls in unserer Vorstellung durch bestimmte geistesgeschichtliche Ereignisse gebildet wird, wie in „Hier manifestiert sich erstmals der Geist der deutschen Romantik in seiner reinsten Form". Allgemein ist ein deiktischer Raum nichts als eine geordnete Menge von Elementen, die als Denotate für die betreffenden Deiktika in Frage kommen. Deiktische Räume unterscheiden sich im wesentlichen durch

a) die Art der Einheiten, die man als Elemente ansieht, z.B. visuelle Objekte, Umgebungen visueller Objekte, eventuell Ereignisse, Passagen in einem Gedankengang (,,hier liegt ein Widerspruch vor" bedeutet etwa soviel wie ,an dieser Stelle im Gedankengang ...") usw.;

b) die Art der Ordnungsbeziehung zwischen diesen Elementen; c) die Zahl der Dimensionen; der Raum der visuellen Wahrnehmung ist nach allgemeiner Auffassung dreidimensional, eine Karte zweidimensional, ,geistesgeschichtliche" Räume sind wahrscheinlich durch eine ganze Reihe von Dimensionen gekennzeichnet usw.; d) die Art der Metrik, falls vorhanden; so ist der geographische Raum in der üblichen Weise metrisiert, wie es sich in Entfernungsangaben: Luftlinie ausdrückt; für den Raum der visuellen Anschauung haben wir gleichfalls ziemlich klare Entfernungsvorstellungen, die sich jedoch durch die übliche euklidische Entfernungsdefinition für dreidimensionale Räume schlecht approximieren lassen ${ }^{1}$; für noch abstraktere Räume haben wir nur noch sehr unklare Entfernungsvorstellungen, die sich schwer metrisieren lassen. Es ist sicher plausibel, den Raum der visuellen Wahrnehmung als ontogenetisch primär anzusehen und alle andern als mehr oder minder abgeleitet, vielleicht auch degeneriert zu betrachten. Dies ändert aber nichts daran, daß wir lokaldeiktische Ausdrücke verwenden, um in abstraktere Räume zu verweisen. Bei der Verwendung muß dabei jeweils sichergestellt werden, daß Sprecher und Hörer über denselben deiktischen Raum verfügen, sonst können sie die jeweiligen deiktischen Ausdrücke nicht sinnvoll interpretieren.

Zur Identifizierung der Denotate von ,hier“, ,da“ usw. genügt es selbstverständlich nicht, daß sich die Sprechenden über den jeweiligen deiktischen Raum einig sind. Sie müssen darin die jeweiligen Teilräume lokalisieren, auf die die betreffenden Ausdrücke in der Situation verweisen. Dazu wird eine Reihe von Techniken angewandt.

Zunächst muß in dem jeweiligen Raum ein Element ausgezeichnet werden, relativ zu dem die Denotate festgelegt werden. Im einfachsten Fall ist dieser primäre Bezugspunkt, die „Origo“ 2 , durch den Sprecher gegeben. Jeder Sprecher bringt gleichsam seine Origo in die Sprechsituation mit. "Hier" verweist dann auf einen Teilraum um den Sprecher, dessen Grenzen offen sind und durch andere sprachliche Mittel markiert werden müssen. Dies setzt natürlich voraus, daß der Sprecher (oder sein Körper) als Element des betreffenden deiktischen Raumes aufgefaßt werden kann. Sonst muß eine Origo explizit eingeführt werden, etwa durch einen sprachlichen Ausdruck. Dies ist z.B. bei ,hier" in Aufsätzen der Fall $(, \ldots$ verweisen wir auf unsere frühere Monographie xyz; hier findet der Leser ..."). Eine Origo-Verschiebung ist allerdings auch in Fällen möglich, in denen der Sprecher sehr wohl ein Element des deiktischen Raumes ist; die „,eigent-

\footnotetext{
Vgl. hierzu die ausgedehnte Literatur über räumliche Wahrnehmung $[8,15]$

2 Dieser Ausdruck ist durch Bühler [2] bekanntgeworden; Bühler verwendet ihn allerdings in einem etwas engeren Sinne als wir hier
} 
liche" Origo geht dann nicht verloren, aber es wird - sehr oft durch Gesten - eine sekundäre Origo eingeführt, die vorübergehend den Bezugspunkt darstellt. Es ist auch zu beachten, daß sich die Position des Sprechers während seiner Äußerung ändern kann, z.B. wenn er durch ein Zimmer geht und sagt ,Von hier sind es genau drei Schritte bis hier". Origo ist in diesem Fall die jeweilige Position des Sprechers. Die Origo-Markierung wirft, falls nicht sekundäre Origines eingeführt werden, für die Kommunikationsteilnehmer insofern ein Problem auf, als zunächst jeder seine eigene Origo in die Sprechsituation einbringt: Der Sprecher bezieht sich mit „hier“", ,dort" usw. auf Teilräume, die u.a. durch seine Origo festgelegt sind; der Hörer hat dies in sein System zu transformieren, denn seine Origo ist ja eine andere. Eine solche Koordination der unterschiedlichen Perspektiven kann in einfachen Fällen durch Zuordnungen wie das Sprecher-Hier ist ein Hörer-Dort, ein Sprecher-Dort ist das Hörer-Hier,

Sprecher-Hier und Hörer-Hier sind meist verschieden, können sich aber überlappen, gelegentlich auch übereinstimmen (je nachdem, wie groß die Teilräume um die beiden Origines sind)

beschrieben werden. Eine nähere Betrachtung würde allerdings zeigen, daß die Zuordnungen noch wesentlich komplexer sind.

Durch die Festlegung der Origines und ihre Koordination ist immer noch nicht sichergestellt, daß die Teilräume, auf die sich die Deiktika beziehen, identifiziert werden können. Das Denotat von „hier“" ist ja nicht die Origo des jeweils Sprechenden, sondern ein Teilraum, der diese Origo enthält. Er kann unterschiedlich weit reichen, und dies wird gleichfalls durch den Kontext der betreffenden Äußerung festgelegt. Dabei folgen wir bestimmten Prinzipien. „Hier" kann sich auf den Stuhl beziehen, auf dem ich sitze, auf das Zimmer, in dem ich bin, auf die Sprache, die Stadt usw.; es ist aber sehr unwahrscheinlich, daß sich ein ,hier" auf den Stuhl und den ihn umgebenden Raum im Abstand von $34,9 \mathrm{~cm}$ bezieht oder auf das Zimmer und die beiden angrenzenden - es sei denn, diese Zimmer bilden wiederum eine Einheit, z.B. ein Seminar. Vielfach wird die Abgrenzung durch sprachliche Mittel gestützt - wie etwa „Hier auf diesem Bett starb Napoleon“ oder „Hier im Himmel wird zuviel gesungen". In anderen Fällen rekonstruieren wir die Grenzen des Hier durch unser Weltwissen: Wenn jemand sagt: „Man liegt hier sehr bequem“, dann sagt uns unser allgemeines Wissen, da $B$ mit „hier" nicht Europa gemeint ist, und umgekehrt in „Hier gibt es keine Gerechtigkeit" nicht ein Bett.

Mit der Abgrenzung sind die Voraussetzungen gegeben, um zumindest das Denotat von ,hier" zu identifizieren: Es verweist auf einen Teilraum des betreffen- den deiktischen Raumes, der innerhalb bestimmter Grenzen liegt und die Origo umfaßt. Es bleibt das Problem, das Denotat eines bestimmten ,dort" und „,da“ zu identifizieren. Beide stehen in einer Opposition zu „,hier": Sie beziehen sich auf einen Teilraum des deiktischen Raums, der nicht die jeweilige Origo als Element enthält. Aber es kann natürlich viele solcher Teilräume geben, ganz abgesehen davon, daß sich auch hier das Abgrenzungsproblem stellt: Beide Probleme werden wiederum durch besondere Mittel gelöst, etwa durch Gesten, durch zusätzliche sprachliche Angaben (,dort wo die Zitronen blühen"); über diese Mittel im einzelnen sowie über die Oppositionen, insbesondere die zwischen , ,dort " und ,da", ist noch wenig bekannt.

Damit haben wir im wesentlichen alle Faktoren zusammen, die die Funktion der lokalen Deixis bestimmen. Dieses System ist ziemlich komplex. Es wird noch komplexer, wenn man die indirekte oder ,, analogische" Deixis berücksichtigt. Damit sind Fälle wie die folgenden gemeint: Wenn jemand auf seine Schulter deutet und sagt: „Die Kugel traf ihn hier“, so meint er natürlich nicht die Stelle an seiner Schulter, sondern die analoge Stelle an der Schulter des andern. Oder wenn jemand auf einen roten Fleck auf einer Karte zeigt und sagt: „Hier wohne ich“, meint er natürlich nicht den roten Fleck, sondern ein Analogon in der Wirklichkeit. Dabei erfolgt also eine unmittelbare Deixis in einen deiktischen Raum („Bildraum"), der einem andern deiktischen Raum oder einem Teil eines solchen Raumes (,Urbildraum“) durch eine Abbildung zugeordnet ist. Man bezieht sich dann auf einen Teilraum des Urbildraums, indem man auf den zugeordneten des Bildraums verweist.

Es ist erstaunlich, daß ein so komplexes System so gut funktioniert, denn in der Praxis haben wir kaum Schwierigkeiten zu identifizieren, was jemand meint, wenn er ,hier", ,da" oder ,dort" sagt. Es ist aber ein sehr naheliegendes System. Seinen Körper, seine Art zu sehen, zu hören, zu tasten, bringt jeder mit, und die menschlichen Erfahrungen, die es uns erlauben, in unserer Vorstellung komplexe, abstrakte Räume aufzubauen und uns darin zu orientieren, sind innerhalb einer Kommunikationsgemeinschaft sehr ähnlich; vielleicht sind viele darunter überhaupt anthropologisch universal. Anderseits legt es die Komplexität des Systems, der Zwang, den jeweiligen deiktischen Raum klarzumachen, Koordinationen vorzunehmen usw., nahe, stabile, von der jeweiligen Kommunikationssituation unabhängige Bezugssysteme zu entwickeln; solche Systeme haben wir für viele Bereiche, z.B. Längen- und Breitenangaben oder für die temporale Deixis die Kalenderzeit. Dies sind jedoch in der menschlichen Kommunikation sekundäre Systeme, die in mancher Beziehung stabiler und einfa- 
cher, aber dafür weniger flexibel sind und nicht so tief in die Funktionsweise der menschlichen Kommunikation hineinreichen wie die deiktischen.

\section{Deixis in Wegauskünften}

Ein Fall, bei dem lokale Deiktika eine eminente Rolle spielen, sind Wegauskünfte. Über sie wurde in dem Nijmegener Projekt daher eine Pilotstudie durchgeführt, deren Ergebnisse inzwischen vorliegen [12] Einige davon sollen hier kurz skizziert werden. Die Studie beruht auf 40 Wegauskünften in Frankfurt/M., bei denen Studenten auf der Zeil (Frankfurts Hauptgeschäftsstraße) nach der alten Oper bzw. nach dem Goethehaus fragten; die Erklärungen wurden heimlich aufgenommen und bilden in transkribierter Form die Datengrundlage. Die Verwendung der Deiktika steht, wie die Analyse ergab, in engem Zusammenhang mit den Prinzipien, nach denen ein Sprecher seine Wegerklärung organisiert, falls er überhaupt dazu in der Lage ist. Er muß sich zunächst einmal in der Gegend auskennen, d.h. er muß über eine ,kognitive Karte" der betreffenden Gegend verfügen grob gesagt, eine komplexe Vorstellung vom Verlauf der Straßen, vom Aussehen der Gebäude und Plätze, von Entfernungen usw. ${ }^{3}$ Diese kognitive Karte ist oft sehr selektiv, gelegentlich sogar falsch; sie braucht auch nicht am Anfang der Erklärung vollständig präsent zu sein, sondern sie kann schrittweise durch „Vergegenwärtigung“ einzelner Züge aufgebaut werden. Auf dieser kognitiven Karte muß der Erklärende nun Standort und Ziel lokalisieren. Die eigentliche Wegerklärung hat nun die Form einer, ,imaginären Wanderung" vom Standort zum Ziel, bei der jeweils markante Punkte aus der kognitiven Karte ausgewählt werden. Relativ zu diesen „Fixpunkten“ werden dann bestimmte Handlungen angegeben, die der Fragende zu vollziehen hat, wenn er an das Ziel kommen will. Diese Fixpunkte, die mit dem Standort anfangen und mit dem Ziel enden, bilden die Bezugsgröße für eine Reihe deiktischer Ausdrücke. Was diese Vorgehensweise unter Aspekten der Deixis so interessant macht, sind besonders die folgenden Punkte:

1. Sprecher und Hörer verfügen zu Anfang nicht über den selben deiktischen Raum; die lokalen Deiktika verweisen zwar stets auf Objekte der visuellen Anschaung; aber zumindest der Fragende, also einer der beiden Kommunikationspartner, muß sich die jeweiligen deiktischen Räume der visuellen Anschauung erst „,erwandern“: Der Antwortende, also jener, der die

\footnotetext{
Der Begriff der "Cognitive map" stammt von dem Psychologen Tolman. Es gibt eine Fülle von Untersuchungen über die Struktur solcher kognitiver Karten [7, 9, 16, 19]
}

Erklärungen gibt, durchwandert sie im Geiste, d.h. er stellt sich bestimmte Areale - das Aussehen der Häuser, der Plätze usw. - vor, wählt Elemente daraus aus und bezieht sich mit Deiktika darauf: ,... bis Sie an den Platz kommen, wo ein großer Brunnen ist, da gehen Sie links runter". Sprecher und Hörer teilen lediglich die Erwartung, daß es sich um den (natürlich ständig wechselnden) Raum der visuellen Wahrnehmung handelt.

2. Die lokaldeiktischen Ausdrücké werden ständig von temporaldeiktischen durchkreuzt, deren Bezugspunkte durch die Sequenz der Handlungen, die zu vollziehen sind, geliefert werden: ,Sie gehen jetzt die Hauptstraße hier an der Biegung geradeaus, und dann ist es die erste Querstraße zur linken Seite“. ,Jetzt" verweist auf die erste Handlung, ,dann" auf den Zeitpunkt, nachdem die erste Handlung abgeschlossen ist; ,erste" bezieht sich auf eine Handlung und auf einen festen Bezugspunkt, in diesem Falle den Ausgangspunkt. Wie das Beispiel zeigt, sind die beiden Typen deiktischer Ausdrücke oft eng miteinander verschränkt. So ist oft auch nicht zu entscheiden, ob „,da" temporal oder lokal zu verstehen ist.

3. Die Fixpunkte werden oft mit Hilfe deiktischer Ausdrücke eingeführt; typische Beispiele dafür sind deiktische Ausdrücke wie ,,erste“, ,nächste“, ,,andere". Es kann sein, daß es überhaupt nur einen einzigen festen Punkt gibt, nämlich den Standort, und alles andere unter Bezug auf diesen Punkt eingeführt wird. Dabei braucht dieser erste Bezugspunkt nicht einmal explizit genannt zu sein. Ein Beispiel dafür ist die vollständige Wegerklärung: „Geh'n Sie da rauf, immer geradeaus, erste Straße links, erste Straße rechts."

4. Streng genommen sind es oft nicht die Fixpunkte allein, die festlegen, was jeweils ein deiktischer Ausdruck bedeutet. Typisch ist die Verwendung von Ausdrücken wie ,links", ,,rechts“" in Wegbeschreibungen. Was links und rechts ist, hängt im wesentlichen von der Blickrichtung des Sprechers ab. In Wegbeschreibungen hängt die Bedeutung dieser Ausdrücke von einer hypothetischen Blickrichtung an einem hypothetischen Standort $a b$, und der Fixpunkt dient dazu, diesen Standort zu fixieren. In den Texten, die in der Pilotstudie untersucht wurden, finden sich Beispiele dafür, daß dieselbe Lokalität, eine Straße, an demselben Fixpunkt, einem Gebäude, einmal als links und einmal als rechts bezeichnet wird (die folgenden Beispiele sind etwas vereinfacht): (a) ,hier vor bis zum Kaufhof, und da halten Sie sich rechts, geradeaus durch die Freßgass“; (b) ,,jetzt geh'n Sie vor, bis ganz vorn hin, bis Sie an den Kuafhof stoßen, dann geh'n Sie links rein, die Freßgass". Der Sachverhalt wird deutlich, wenn man sich anhand eines Kartenausschnitts (Fig. 1) die Situation vergegenwärtigt: Im 


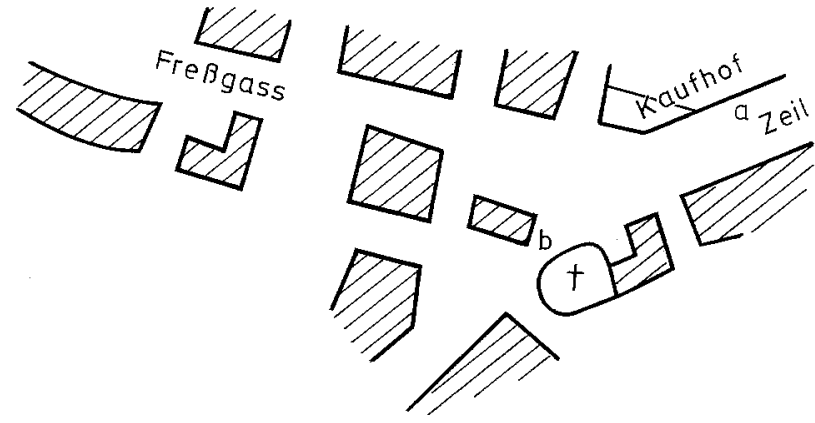

Fig. 1

ersten Fall steht der Wegerklärende an Punkt (a); wenn der Fragende von dort in Richtung Kaufhof geht, entspricht seine Blickrichtung einer Linie von (a) zum Kaufhof, und am Kaufhof liegt die Straße dann rechts; im zweiten Fall wird die Beschreibung an Punkt (b) gegeben, und vor dem Kaufhof entspricht seine Blickrichtung normalerweise einer Linie von (b) zu seiner hypothetischen Position am Kaufhof; dann liegt die Freßgasse links.

Dies ist ein markantes Beispiel, wie räumliche Orientierung - also ein durchaus nichtsprachlicher Faktor - nach festen Regeln determiniert, worauf ein bestimmter Ausdruck in einer bestimmten Situation verweist. Wenn man eine realistische Vorstellung davon gewinnen will, wie die Sprache tatsächlich funktioniert, muß man solche Faktoren mit berücksichtigen.

\section{Ellipse}

\section{Probleme regulärer Ellipsen}

In einer Szene von „Werthers Leiden“ stehen Lotte und Werther am Fenster, schauen in den milden Regen nach einem Gewitter, und ,sie legte ihre Hand auf die meinige und sagte - Klopstock!" . Diese ÄuBerung ist hochgradig elliptisch; beide verstehen aber genau, was gemeint ist. Es wird schwer halten, Regeln dafür anzugeben, denen zufolge die Äußerung in dieser Situation genau das bedeutet, was Lotte und Werther verstehen; die Ellipse ist idiosynkratisch. Von regelhaften Ellipsen kann man sprechen, wenn der Sprecher bei seiner Äußerung bestimmte Elemente weglassen kann, die der Hörer nach festen Regeln aus dem Kontext zu ergänzen vermag. Die Äußerung „im Garten“ als Antwort auf die Frage „Wo ist morgen abend die Party?" wird regelhaft im Sinne von „Die Party ist morgen abend im Garten“ verstanden. Als Antwort auf die Frage „Wo lag denn der
Schlauch?" bedeutet sie regelhaft „,der Schlauch lag im Garten", und niemand, der Deutsch kann, wird dies anders verstehen.

Damit eine Einheit regelhaft aus dem Kontext ergänzt werden kann, ist es gewöhnlich erforderlich, daß sie nach bestimmten Prinzipien in den Kontext eingeführt wurde. Dies kann beispielsweise durch ,,parallele Wahrnehmung" von Sprecher und Hörer in einer Situation geschehen. Wenn etwa beide auf dem Sportplatz gerade einen Fallrückzieher gesehen haben und einer der beiden sagt ,toll“", so meint der damit ,,der Fallrückzieher war toll“ und nicht, ,das Leben ist toll", weil er davon ausgehen kann, daß der andere auf dasselbe Objekt (bzw. Ereignis) fokussiert ist. Dies ist ein üblicher Fall von Ellipse, aber die Prinzipien, denen er folgt - und die sicherstellen, daß vom gleichen Thema die Rede ist -, sind schwer zu ermitteln. Einfacher ist es, wenn die weglaßbaren Einheiten sprachlich in den Kontext eingeführt werden. Dann wird nämlich nicht nur explizit gesagt, worum es geht, sondern es werden zugleich syntaktische Informationen vermittelt. Es kann z.B. sein, daß ein Thema in einem ersten Satz in Subjektstellung eingeführt wird; will man dann über dasselbe Thema noch etwas sagen, so braucht es nicht mehr genannt zu werden, wenn es wieder in Subjektstellung stehen soll: ,Der Vater liebt die Mutter und (der Vater) haßt die Tantec. Es werden also nicht nur semantische, sondern auch syntaktische Informationen bezüglich Subjekt, Kasus, Numerus u.v.a. im Arbeitsgedächtnis eingespeichert, die dann bei der Produktion wie bei der Interpretation des zweiten, elliptischen Teiles verfügbar sind. Eine zentrale Frage ist es nun, welche Informationen nicht mehr eigens angegeben werden müssen. Wenn jemand fragt: „Was hat Fritz gemacht?“", so führt er damit eine Information ein, etwa, daß es um Fritz geht (Subjekt) und daß die Handlung in der Vergangenheit liegt. Als Antwort genügt,,geschlafen" im Sinne von „Fritz hat geschlafen“; man kann nicht auf die erneute Angabe verzichten, daß sich die Handlung in der Vergangenheit vollzogen hat, und einfach sagen ,,schlafen", obwohl auch dies schon klar ist. Es gibt hier offenbar feste Regeln, über die man erst wenig weiß, die zu untersuchen aber wesentlich ist, wenn man ein angemessenes Bild davon gewinnen will, wie sprachliche Verständigung funktioniert. Ellipsen dieser Art sind keine Ausnahmefälle. Sie sind ein zentrales Kennzeichen des sprachlichen Verhaltens.

Im folgenden werden einige Ergebnisse einer Pilotstudie referiert, bei der untersucht wurde, welche Informationen gespeichert und dann weggelassen werden. ${ }^{4}$

\footnotetext{
4 Eine ausgedehnte Analyse syntaktischer Bedingungen für die Verwendung von Ellipsen in koordinierten Sätzen - nicht in Frage-Antwort-Folgen - findet sich in [13]
} 


\section{Die Produktion elliptischer Antworten}

Es ist noch völlig unklar, welche psychologischen Mechanismen der Produktion elliptischer Antworten zugrundeliegen. Das empirische Problem, das es zu lösen gilt, läßt sich am besten durch eine Gegenüberstellung zweier extremer theoretischer Positionen klarmachen. Angenommen, jemand soll die Frage ,Wem hat Fritz das Buch geschickt?" beantworten. Dafür sind zwei Prozeduren denkbar; die erste wollen wir „,syntaktisch“, die zweite ,semantisch" nennen. Bei der syntaktischen durchläuft der Sprecher folgende Stadien:

(1) Speichere die Frage im Kurzzeit-Gedächtnis (so wie man eine gerade gehörte Telefonnummer lange genug im Kurzzeit-Gedächtnis speichert, um sie zu wählen).

(2) Bringe die Frage in Antwort-Format: „Fritz hat $\mathrm{X}$ das Buch geschickt" und bilde einen semantischen Kode dafür, den man etwa in der Form Schicken (Fritz, Buch, X) darstellen kann.

(3) Behalte das Antwort-Format im Kurzzeit-Gedächtnis und verwende den semantischen Kode, um im Langzeit-Gedächtnis nach der Information $X \mathrm{zu}$ suchen (das Langzeit-Gedächtnis ist unser Speicher für permanentes oder halb-permanentes Wissen, wie etwa das unserer eigenen Telefonnummer). Angenommen nun, die gefundene Information sei „Peter".

(4) Nimm die gefundene Information ins KurzzeitGedächtnis und setze sie an die Stelle von X.

(5) Tilge alles außer ebendieser Information (nämlich Peter) und äußere sie.

Diese syntaktische Prozedur liefert die Antwort „Peter", aber auf den ersten Blick scheint sie eine weit abführende und unökonomische Weise, die Antwort zu erzeugen. Das zweite Stadium scheint überflüssig; es scheint nicht sonderlich sinnvoll, das Antwort-Format insgesamt zu erzeugen und $\mathrm{zu}$ speichern, wenn der größte Teil davon in Stadium (5) dann wieder gelöscht wird.

Die zweite, semantische Prozedur läuft wie folgt:

(1) Speichere die Frage im Kurzzeit-Gedächtnis.

(2) Bilde einen semantischen Kode dafür, d.h.. Schikken (Fritz, Buch, X).

(3) Lösche den Wortlaut der Frage im Kurzzeit-Gedächtnis und suche mithilfe des semantischen Kodes im Langzeit-Gedächtnis nach der X entsprechenden Information.

(4) Nimm die Information ins Kurzzeit-Gedächtnis und äußere sie.

Auch hier ist die Antwort „Peter", wenn alles glatt läuft. Hier wird die syntaktische Information im Kurzzeit-Gedächtnis gelöscht, sobald die semantische Information vorliegt; ein Tilgungsproze $\beta$ ist nicht erforderlich. Auf den ersten Blick scheint dies ökonomi- scher, aber das kann täuschen. In manchen Fällen zumindest wäre es ökonomischer, zumindest einen Teil der syntaktischen Information während des semantischen Suchlaufs im Arbeitsgedächtnis verfügbar zu halten. Man betrachte die oben diskutierte Frage „Was hat Fritz gemacht?". Offenbar hat die angemessene Antwort ,geschlafen“ dieselbe syntaktische Form wie ,gemacht". Während des semantischen Suchlaufs arbeitet der Sprecher mit der Information „schlafen in der Vergangenheit", aber das genügt nicht, um die Form ,geschlafen" festzulegen; es könnte auch zu der Form ,schlief“ führen. Der Sprecher übernimmt aber die Form offenbar aus der Frage, und dies legt die Annahme nahe, daß er sie während des semantischen Suchlaufs im Kurzzeit-Gedächtnis behält. Nun ist jedoch Information im Kurzzeit-Gedächtnis sehr anfällig. Sie wird leicht durch neu hinzukommende Information gelöscht, so daß diese Prozedur nur effizient ist, wenn das KurzzeitGedächtnis relativ wenig belastet wird.

Derzeit laufen verschiedene Experimente im Nijmegener Projekt, mit deren Hilfe geklärt werden soll, wie der semantische Suchlauf und die unmittelbare Speicherung des Wortlautes und der syntaktischen Form während des Frage-Antwort-Prozesses zusammenwirken. Es sind größtenteils Laborexperimente mit genauer Aufzeichnung der Antwort, der Reaktionszeit usw. Ziel eines kürzlich abgeschlossenen Experimentes war es, die Rolle des syntaktischen Kodes im Wortlaut vorzuführen, und zwar sollte nachgewiesen werden, daß

(a) rein syntaktische Aspekte der Frage die syntaktische Form der Antwort beeinflussen,

(b) diese Wirkung verlorengeht, wenn die Frage ein $\mathrm{zu}$ hohes Maß an Zusatzinformationen enthält; diese Information nämlich löscht den syntaktischen Kode aus dem Kurzzeit-Gedächtnis.

Man vergleiche das Beispiel ,Wem hat Fritz das Buch geschickt?" mit "An wen hat Fritz das Buch geschickt?". Man kann in der Praxis annehmen, daß beide Fragen semantisch äquivalent sind. Die rein semantische Prozedur müßte daher zum gleichen Antwort-Format führen. Läßt sich hingegen eine Tendenz zu verschiedenen Antworten nachweisen (z.B. mehr Antworten ,Peter" im ersten und ,an Peter" im zweiten Fall), dann ist damit belegt, daß Elemente des Frage-Formats während des semantischen Suchlaufs im Gedächtnis behalten werden.

Handelt es sich dabei um das Kurzzeit-Gedächtnis, so müßte es leicht durch Zusatzinformationen für den Höhrer zu beeinflussen sein, etwa indem man fragt „Wem (bzw. ,an wen") hat Fritz das Buch geschickt, weißt du, das Buch, das er letzte Woche gekauft hat und von dem er dauernd erzählt?" Dies reicht vielleicht, um die Spuren des syntaktischen Formates aus- 
zulöschen, so daß kein Unterschied auf die beiden semantisch äquivalenten Fragen hin mehr zu erkennen ist. In dem Experiment haben wir daher semantisch äquivalente kurze Fragen mit und ohne Präposition sowie semantisch äquivalente lange Fragen mit und ohne Präposition verwendet.

In Nijmegen herrscht allgemein Unklarheit darüber, wann die Geschäfte am Samstag schließen. Dies bot sich für ein Experiment an: An aufeinanderfolgenden Samstagen riefen wir hintereinander 160 Geschäfte in der Stadt an und stellten eine der folgenden Fragen (wir geben eine behelfsweise Übersetzung der Fragen; der Effekt läßt sich im Deutschen nicht genau kopieren):

(1) Hoe laat gaat uw winkel dicht? (kurze Frage ohne Präposition) (Wann geht Ihr Geschäft zu?)

(2) Om hoe laat gaat uw winkel dicht? (kurze Frage mit Präposition) (Um wann geht Ihr Geschäft zu?)

(3) Hoe laat gaat uw winkel dicht, want ik moet er extra voor naar de stad komen, ziet $\mathrm{u}$ ? (lange Frage ohne Präposition) (Wann geht Ihr Geschäft $\mathrm{zu}$, denn ich muß extra deswegen in die Stadt kommen, wissen Sie?)

(4) Om hoe laat gaat uw winkel dicht, want ik moet er extra voor naar de stad komen, ziet $u$ ? (lange Frage mit Präpostion)

Die Antworten wurden gleichzeitig von zwei Hörern aufgezeichnet. Wir sagten voraus, daß Frage (1) weniger Antworten mit Präposition zu Folge haben würde als Frage zwei, d.h. eher Antworten wie ,vijf uur" (,,ünf Uhr") als ,om vijf uur" (,,um fünf Uhr"); zwischen den Antworten auf (3) und (4) erwarteten wir keinen Unterschied. Und ebendies ergab sich. Die Antworten auf (3) waren zudem weit weniger elliptisch als die auf (1). Für das Vorkommen elliptischer Antworten scheint es eine Bedingung zu sein, daß die Fragesatzinformation noch frisch im Gedächtnis steht.

Natürlich sind auch alternative Interpretationen dieser Ergebnisse denkbar. Man könnte z.B. argumentieren, es bestehe eine soziale Konvention, ,je länger die Frage, desto weniger elliptisch die Antwort" damit wäre das Arbeitsgedächtnis nicht für diese Er- gebnisse verantwortlich. Dies läßt sich nur durch weitere Experimente klären, aber selbst wenn eine solche soziale Konvention besteht, ergibt sich die Frage, welchen Sinn sie hat. Viele soziale Konventionen haben die Funktion, den Informationsaustausch angesichts der bestehenden biologischen Beschränkungen für die Informationsverarbeitung in den Individuen zu optimieren. Die Konventionen für Ellipse bei Frage und Antwort könnten ein Beispiel dafür sein. Dies kann letztlich nur durch sorgfältige weitere Forschungen geklärt werden.

1. Baddeley, A.D., Hitsch, G., in: The Psychology of Learning and Motivation, Vol. 8 (G.H. Bower, ed.). 1974

2. Bühler, K.: Sprachtheorie. Jena 1934

3. Eigen, M., Winkler-Oswatitsch, R.: Das Spiel, Kap. 15. München: Piper 1975

4. Ertel, S., in: Sentence Production: Developments in Research and Theory (S. Rosenberg, ed.). Washington: Erlbaum 1977

5. Fillmore, Ch.: Found. Lang. 2, 219 (1966); Santa Cruz Lectures on Deixis, 1971. Indiana Univ. Linguistic Club 1975

6. Fillmore, Ch., in: Semantik und generative Grammatik I, S. 147 (F. Kiefer, ed). Frankfurt 1972

7. Hardwick, D.A., McIntyre, C.W., Pick, H.L.: Monogr. Soc. Res. Child Dev. 166, 41 (1976)

8. Indow, T.: Psychologia 17, 50 (1974)

9. Kaplan, S., in: Image and Environment (R.M. Downs, D. Stea, eds.). Chicago: Aldine 1973

10. Kempen, G.: Psychology of Language Conf. 1977

11. Klein, W.: Wo ist hier? Präliminarien zu einer Untersuchung der lokalen Deixis. Max-Planck-Gesellschaft, Projektgruppe für Psycholinguistik, Nijmegen 1977

12. Klein, W.: Wegauskünfte. Max-Planck-Gesellschaft, Projektgruppe für Psycholinguistik, Nijmegen 1977

13. Klein, W.: Reguläre Ellipsen im Deutschen. Max-Planck-Gesellschaft, Projektgruppe für Psycholinguistik, Nijmegen 1977

14. Labov, W., Linde, Ch.: Language 51, 924 (1975)

15. Luneburg, R.K.: J. Opt. Soc. Am. 40, 637 (1950)

16. Lynch, K.: The Image of a City. Cambridge, Mass.: M.I.T. 1960

17. Lyons, J.: Deixis as the Source of Reference. L.A.U.T. 1973

18. Osgood, C.E., in: Sentence Production: Developments in Research and Theory (S. Rosenberg, ed.). Washington: Erlbaum 1977

19. Tolman, E.C.: Psychol. Rev. 55, 189 (1948)

20. Wunderlich, D.: LiLi 1/2, 153 (1971)

Eingegangen am 15. März 1978 